Accepted for publication in Astrophysics and Space Science

\title{
Topical Issues for Particle Acceleration Mechanisms in Astrophysical Shocks
}

\author{
Matthew G. Baring \\ Department of Physics and Astronomy MS-108, Rice University, \\ P.O. Box 1892, Houston, TX 77251, U.S.A. baring@rice.edu
}

\begin{abstract}
Particle acceleration at plasma shocks appears to be ubiquitous in the universe, spanning systems in the heliosphere, supernova remnants, and relativistic jets in distant active galaxies and gamma-ray bursts. This review addresses some of the key issues for shock acceleration theory that require resolution in order to propel our understanding of particle energization in astrophysical environments. These include magnetic field amplification in shock ramps, the non-linear hydrodynamic interplay between thermal ions and their extremely energetic counterparts possessing ultrarelativistic energies, and the ability to inject and accelerate electrons in both non-relativistic and relativistic shocks. Recent observational developments that impact these issues are summarized. While these topics are currently being probed by astrophysicists using numerical simulations, they are also ripe for investigation in laboratory experiments, which potentially can provide valuable insights into the physics of cosmic shocks.
\end{abstract}

\section{Introduction}

Supersonic flows abound in the cosmos, as do emission regions exhibiting non-thermal radiation. The intimate connection between the two establishes that particle acceleration in astrophysical shocks is germane to many systems, ranging from the heliosphere, to stars of various sorts expelling winds, to supernova remnants, to extragalactic jets and gamma-ray bursts. In the case of heliospheric shocks such as travelling interplanetary discontinuities and planetary bow shock environs, we can immerse ourselves in the plasma experiment via in situ spacecraft measurements of non-thermal ions, electrons and turbulent magnetic fields. While localized and therefore sparse in terms of the spatial sampling, these observations do provide profound insights into the complexity of the shock acceleration phenomenon. In astrophysical sites beyond the solar system, our role is passive, as observers of signals from 
remote sites of acceleration. Moreover, the information on plasma properties is subject to a convolution with radiative processes, complicated source morphology within our spatial resolution scale, and propagational modification along the line of sight to sources.

Observationally, radio, optical and X-ray telescopes have provided groundbreaking insights into the shock acceleration phenomenon, due to advances in the angular resolution and spectral sensitivity. In addition, the gamma-ray field is generating a greater understanding of source energetics as we find that many non-thermal astronomical sources emit most of their power in the gamma-rays. Progress on the theoretical front builds on the observational advances, and has turned more to computer simulations due to the dramatically enhanced speed of computers over the last two decades. Yet astrophysical code verification is an increasingly salient issue as their complexity and computational demands burgeon. This provides a niche for laboratory plasma experiments that are tailored for the problem of astrophysical particle acceleration. Supersonic flows can be generated in controlled environments, and work along these lines focuses naturally on using lasers to mimic blast waves and jets, supernovae and supernova remnants (e.g. Borovsky et al. 1984; Drake et al. 1998; Shigemori, et al. 2000; Kang et al. 2001; Lebedev et al. 2002; Woolsey, Courtois \& Dendy 2004), and probe hydrodynamic and magnetohydronamic aspects. The question of scalability of such laboratory findings to astrophysical systems (e.g. Ryutov et al. 2001; see also the review of Remington, Drake \& Ryutov 2006) is obviously of central importance. Here, an offering on some topical issues for particle acceleration at astrophysical shocks is made, to provide a basis for the community in high energy density plasma physics to help identify germane astrophysical problems that might be well suited for interdisciplinary investigation.

\section{Magnetic Field Enhancements in Shocks}

One of the key properties of shock structure that is germane to the acceleration of high energy cosmic rays is the strength of the magnetic field $B$ near the shock. In astrophysical shocks this cannot really be measured directly, since there is generally a lack of viable spectral line diagnostics: the Zeeman effect and cyclotron emission/absorption features are generally broadened, small or non-existent in diffuse, turbulent shock environs. Normally, proximity of an emission region to a stellar surface, such as in white dwarfs and neutron stars is required to afford precise magnetic field measurements. In heliospheric shocks, magnetometer data discern the chaotic nature of pre- and post-shock fields, and a prominent property appears to be (e.g. Baring et al. 1997) a shock-induced compression of the field in the downstream region that is fairly close to magnetohydrodynamic (MHD) determinations that are derived from momentum and energy flux conservation across the shock, i.e. the 
so-called Rankine-Hugoniot conditions (e.g. Drury 1983; Jones \& Ellison 1991). If the interaction of charged particles with shock-associated field turbulence is gyroresonant at the Doppler-shifted cyclotron frequency (e.g., see Melrose 1980), as is expected for Alfvén and whistler modes, then the acceleration timescale $\tau$ naturally scales as the gyroperiod (i.e., $\tau \sim 1 / \nu_{g} \propto 1 / B$; see Forman, Jokipii \& Owens 1974; Drury 1983) and the corresponding diffusive lengthscale is comparable to the Larmor radius $r_{g}(\propto 1 / B)$. Hence the magnitude $B$ establishes the temporal, spatial and energy scales of acceleration at a shock, and so is a critical parameter for the energization process.

Since the general paradigm of galactic cosmic rays (CRs) invokes supernova remnants (SNRs) as the sites for their production (see Drury 1983 for a review), knowledge of the field strength in proximity of their shocks is vital. Directional information can be obtained on fairly large spatial scales via radio synchrotron polarization data (e.g. see Rosenberg 1970; Downs \& Thompson 1972; Anderson, Keohane \& Rudnick 1995, for Cassiopeia A), but values of $B=|\mathbf{B}|$ are not forthcoming. Estimates for the field strength can be inferred by modeling the continuum flux level in a given wavelength band, but these are subject to a number of assumptions about the medium, for example the mean density $n_{e}$ and the Lorentz factors $\gamma_{e}$ of the radiating electrons. The understanding of the character of shells and interiors of SNRs has recently been advanced by groundbreaking observations with the Chandra X-ray Observatory, enabled by its impressive angular resolution coupled with its spectral capabilities. Of particular interest is the observation of extremely narrow nonthermal $(1.2-2.0 \mathrm{keV})$ X-ray spatial profiles in selected remnants (see Long et al. 2003 and Bamba et al. 2003 for the northeast limb of SN1006; Vink \& Laming 2003 for Cas A; for theoretical modeling see Ellison \& Cassam-Chenaï 2005, and Völk et al. 2005), typically less than 5-10 arcsec across. Upstream of these shell shocks, the X-ray emission, which is thought to be synchrotron in origin, drops to effectively zero. These strong brightness contrasts between the shell, and the outer, upstream zones correspond to flux ratios exceeding $R \gtrsim 50$. The narrowness of profiles along image scans argues for the shocks being aligned perpendicular to the sky, i.e., offering no projectional smearing in the images. Note also that the surface brightness angular profiles in SN1006 and Cas A are much broader for the thermal X-rays $(0.5-0.8 \mathrm{keV})$ and the radio synchrotron than for the non-thermal X-rays.

If the synchrotron mechanism is indeed responsible for non-thermal Chandra emission, the electrons contributing to the Chandra signal are probably in a strongly-cooling regime: see Baring et al. (1999) for a comprehensive discussion of SNR cooling parameter space. Since the synchrotron cooling rate for an electron scales as $\gamma_{e}^{2} B^{2}$, then the flux ratio $R$ is approximately a measure of the ratio of $B^{2}$ downstream $(\mathrm{d})$ to upstream $(\mathrm{u})$. The observed lower bounds to $R$ considerably exceed values $R \lesssim 16$ expected for magnetohydrodynamic compression at the shocked shell; at a plane-parallel shock with $\mathbf{B}$ along the shock normal 
there is no field compression, while in a strong (i.e. high sonic Mach number) perpendicular shock with $\mathbf{B}$ in the shock plane, $B_{d} / B_{u} \sim 4$. Hence, the pronounced brightness contrast is taken as strong evidence of magnetic field amplification in the shock precursor/ramp upstream. Higher fields are obviously advantageous to cosmic ray production issues. Historically-accepted values of $B \sim 1-10 \mu$ Gauss (i.e. $0.1-1$ nanoTesla) are somewhat too small to permit acceleration in SNR shocks of ages around $10^{3}-10^{4}$ years right up to the cosmic ray knee at $\sim 3 \times 10^{15} \mathrm{eV}$ (e.g. see Lagage \& Cesarsky 1983). This problem has spawned the suggestion (Jokipii 1987) that relatively ineffective diffusive transport of particles orthogonal to the mean field direction in quasi-perpendicular regions of SNR shocks can speed up acceleration of ions to higher energies, helping access the knee. Yet, this enhanced rapidity is accompanied by reduced efficiency of cosmic ray injection from thermal energies (Ellison, Baring \& Jones 1995). Hence, truly larger fields provide a cleaner path for acceleration in remnants to reach the cosmic ray knee (e.g. Kirk \& Dendy 2001).

The X-ray observational developments have been accompanied by theoretical proposals of magnetic field amplification in the upstream shock precursor. Most notable has been the work of Lucek \& Bell (2000), and subsequent papers such as Bell (2004), Amati \& Blasi (2006), and Vladimirov, Ellison \& Bykov (2006). The idea of Lucek \& Bell is that high energy cosmic rays in strong shocks could amplify $B$ when streaming upstream, adiabatically transferring energy to the turbulent field by pushing against it, simultaneously decelerating the upstream flow. If this process is efficient, the rate of work done on the upstream Alfvén turbulence of energy density $U_{\mathrm{A}}$ naturally scales roughly with the $\mathrm{CR}$ pressure gradient: $d U_{\mathrm{A}} / d t=v_{\mathrm{A}}\left|\nabla P_{\mathrm{CR}}\right|$. Here $v_{\mathrm{A}}=B / \sqrt{4 \pi \rho}$ is the Alfvén speed, and $P_{\mathrm{CR}}$ is the cosmic ray pressure. The associated field amplification should then scale as $(\delta B / B)^{2} \sim \mathcal{M}_{\mathrm{A}} P_{\mathrm{CR}} / \rho u_{u}^{2}$ in an upstream flow of speed $u_{u}$ and mass density $\rho$; this then becomes very effective for high Alfvénic Mach number (i.e. $\mathcal{M}_{\mathrm{A}} \equiv u_{u} / v_{\mathrm{A}} \gg 1$ ), strong shocks that generate large cosmic ray pressures. While this hypothesis is reasonable, demonstrating it is non-trivial. Various MHD-type simulations have been employed by Bell, such as in Bell (2004), where large-scale currents are used to drive instabilities that amplify the upstream field. The persistence of currents on large scales is unclear, particularly due to the action of Debye screening. Moreover, self-consistent physical connection between the cosmic rays of large Larmor radii and the field turbulence of much shorter wavelengths is extremely difficult to explore with MHD or plasma simulations, due to the wide disparity in spatial scales involved. This is an issue also for a growing number of particle-in-cell (PIC) simulations (e.g. Silva et al. 2003; Hededal et al. 2004; Nishikawa et al. 2005; see Section 4 below) used to explore field enhancement via the Weibel instability in relativistic shocks; such developments are not that salient for the problem of amplifying Alfvén turbulence in non-relativistic shocks, and mostly probe the inertial scales of thermal ions and electrons defined by their plasma frequencies. 


\section{Non-linear Feedback Between the Acceleration and the Hydrodynamics}

Non-relativistic collisionless shocks can be highly efficient accelerators, placing 10-50\% of the bulk flow kinetic energy into non-thermal particles. Evidence from theory, computer simulations, and spacecraft observations supports this conclusion; in particular, see Ellison, Möbius, \& Paschmann (1990), for a study of the Earth's bow shock, and Drury (1983), Blandford \& Eichler (1987), and Jones \& Ellison (1991) for reviews. With such efficiencies, the accelerated particles acquire a sizable fraction of the total energy budget, influencing the shock hydrodynamics, and therefore also the fraction of energy going into accelerated particles, in a non-linear manner. The modified flow velocity spatial profile in the shock deviates from the familiar step-function form in test-particle acceleration scenarios, with the energetic particles pushing against the upstream flow and decelerating it far ahead of the shock discontinuity. Accordingly an upstream shock precursor forms, with declining flow velocity as the shock is approached. This structure alters the shape of the energetic particle distribution from a power-law in momentum (e.g., Eichler 1984; Ellison \& Eichler 1984; Ellison, Baring \& Jones 1996; Berezhko et al. 1996; Malkov 1997; Blasi 2002), the canonical test-particle form where the diffusively-accelerated particle distribution samples no spatial or momentum scale. The index $\sigma=(r+2) /(r-1)$ of this power-law $d n / d p \propto p^{-\sigma}$ is purely a function of the compression ratio $r=u_{u} / u_{d}$ of upstream $\left(u_{u}\right)$ to downstream $\left(u_{d}\right)$ flow speed components normal to the shock in the shock rest frame (e.g. see Drury 1983; Jones \& Ellison 1991), and is independent of the magnetic field orientation or the nature and magnitude of the turbulence effecting diffusive transport in the shock neighborhood.

The spatial variation of the upstream flow in strong shocks that are efficient accelerators eliminates the scale independence. Since the highest energy particles have greater diffusive mean free paths $\lambda$ (generally true for gyroresonant interactions with MHD turbulence, and certainly so near the Bohm diffusion limit $\lambda \sim r_{g}$ ), they diffuse farther into the upstream shock precursor against the convective power of the flow, and therefore sample greater effective velocity compression ratios $r$. Accordingly, they have a flatter distribution, yielding a distinctive concavity to the overall particle spectrum, i.e. $\sigma$ is now a declining function of momentum $p$. These departures from power-law behavior amplify the energy placed in the particles with the greatest momenta, which in turn feeds back into the shock hydrodynamics that modify the spatial flow velocity profile. Traveling discontinuities possessing this complex feedback are termed non-linear shocks, the non-linear label being ascribed to the interplay between the macroscopic dynamics and the microscopic acceleration process. Clearly, the possible magnetic field amplification in the upstream precursor that was discussed in Section 2 contributes to the overall dynamics/energy budget of the magnetohydrodynamic flow, and so intimately influences this non-linear aspect of astrophysical shocks. 
The deviations from power-law distributions obviously impact the radiation signatures produced by these particles, with alterations in the fluxes expected in X-ray and TeV gammaray bands in remnants, differing by as much as factors of 3-10 from traditional test-particle predictions (e.g., see Baring, et al. 1999; Ellison, Slane \& Gaensler 2001; Berezhko et al. 2002; Baring, Ellison \& Slane 2005). Conclusively confirming the existence of this non-linear spectral concavity is a major goal that is inherently difficult, since it demands broad, multiwavelength spectral coverage. There is a limited suggestion of concavity in radio data for Tycho's and Kepler's SNRs (Reynolds \& Ellison 1992), and in a multi-wavelength modeling of SN 1006 (Allen, Houck \& Sturner 2004; see also Jones et al. 2003 for inferences from radio and infra-red data from Cassiopeia A), but this task really looks ahead to the launch of the GLAST gamma-ray mission in late 2007, when, in conjunction with ground-based Atmospheric Cerenkov Telescopes probing the $\mathrm{TeV}$ band, it may prove possible to determine gamma-ray spectra from SNRs spanning over 3 decades in energy.

In the meantime, an interesting astrophysical manifestation of these non-linear effects has been offered by SNR observations by the Chandra X-ray Observatory, looking instead at the thermal populations. Inferences of ion temperatures in remnant shocks can be made using proper motion studies, or more direct spectroscopic methods (e.g. Ghavamian et al. 2003). For the remnant 1E 0101.2-7129, Hughes et al. (2000) used a combination of ROSAT and Chandra data spanning a decade to deduce an expansion speed. Electron temperatures $T_{e}$ are determined by line diagnostics, via both the widths, and the relative strengths for different ionized species. From these two ingredients, Hughes et al. (2000) observed that, in selected portions of the SNR shell, $3 k T_{e} / 2 \ll 3 k T_{p} / 2 \sim m_{p}\left(3 u_{u} / 4\right)^{2} / 2$. Therefore, the electrons were considerably cooler than would correspond to equipartition with thermal protons heated in a strong shock with an upstream flow speed of $u_{u}$ : the thermal heating is assumed comparable to the kinematic velocity differential $u_{u}-u_{d} \approx 3 u_{u} / 4$. The same inference was made by Decourchelle et al. (2000) for Kepler's remnant, and by Hwang et al. (2002) for Tycho's SNR. This property of comparatively cooler electrons may be indicative of them radiating very efficiently radiating. Or it may suggest that the protons are cooler (i.e. $\left.3 k T_{p} / 2 \ll m_{p}\left(3 u_{u} / 4\right)^{2} / 2\right)$ than is widely assumed in the test-particle theory, the conclusion drawn by Hughes et al. (2000) and Decourchelle et al. (2000). This effect is naturally expected in the non-linear shock acceleration scenario: as the highest energy particles tap significant fractions of the total available energy, they force a reduction in the thermal gas temperatures. Such feedback can profoundly influence shock layer thermalization, inducing significant interplay with electrostatic equilibration between low energy electrons and ions, an issue addressed in these proceedings by Baring \& Summerlin (2006). Note that non-linear modifications may vary strongly around the shocked shell of an SNR, since the obliquity angle $\Theta_{\mathrm{Bn}, \mathrm{u}}$ of $\mathbf{B}$ to the shock normal varies considerably between different rim locales. 


\section{The Character of Relativistic Shocks}

Relativistic shocks, for which the upstream flow Lorentz factor $\gamma_{u}=1 / \sqrt{1-\left(u_{u} / c\right)^{2}}$ considerably exceeds unity, are less well researched than their non-relativistic counterparts, not in small part due to their greater cosmic remoteness: they predominantly arise in extragalactic locales like jets in active galaxies, and gamma-ray bursts. Yet, because of such associations, they are now quite topical. Diffusive test-particle acceleration theory in parallel, (i.e., $\Theta_{\mathrm{Bn}, \mathrm{u}}=0^{\circ}$ ) relativistic shocks identifies two notable properties in such systems: (i) particles receive a large energy kick $\Delta E \sim \gamma_{u} m c^{2}$ in their first shock crossing (e.g., Vietri 1995), but receive much smaller energy boosts for subsequent crossing cycles (factors of around two: e.g., Gallant \& Achterberg 1999; Baring 1999); (ii) a so-called 'universal' spectral index, $\sigma \sim 2.23$ exists in the two limits of $\gamma_{u} \gg 1$ and small angle scattering, i.e., $\delta \theta \ll 1 / \gamma_{u}$ (e.g., Kirk et al. 2000; see also Bednarz \& Ostrowski 1998; Baring 1999; Ellison and Double 2004). Here, $\delta \theta$ is the average angle a particle's momentum vector deviates in a scattering event, i.e. an interaction with magnetic turbulence.

These characteristics are modified in parallel, mildly relativistic shocks with $\gamma_{u} \sim 1$. In such shocks, the distribution $d n / d p$ remains a power-law (scale-independence persists), but hardens ( $\sigma$ decreases) as either $\gamma_{u}$ drops, or the scattering angle, $\delta \theta$, increases (e.g., Ellison, Jones \& Reynolds 1990; Baring 1999; Ellison \& Double 2004; Baring 2004), even if the compression ration $r=u_{u} / u_{d}$ is held constant (it usually increases with declining $\gamma_{u}$ due to a hardening of the Jüttner-Synge equation of state). These effects are consequences of large kinematic energy kicks particles receive when scattered in the upstream region after transits from downstream of the shock. It is particularly interesting that when scattering conditions deviate from fine pitch-angle-scattering regimes with $\delta \theta \ll 1 / \gamma_{u}$, the power-law index is dependent on $\delta \theta$, with a continuum of spectral indices being possible (Ellison \& Double 2004; Baring 2004). Then the nature of the turbulence is extremely influential on the acceleration outcome, so that understanding the turbulence is of paramount importance. This sensitivity of $\sigma$ to the field fluctuations when $\delta \theta \gtrsim 1 / \gamma_{u}$, a large angle scattering domain, contrasts the canonical nature of $\sigma$ in non-relativistic shocks mentioned above.

In jets and gamma-ray bursts, ultra-relativistic shocks are typically highly oblique due to the Lorentz transformation of ambient, upstream magnetic fields to the shock rest frame. This introduces an added dimension of variation, with increasing $\Theta_{\mathrm{Bn}, \mathrm{u}}$ dramatically steepening the power-law, i.e. increasing $\sigma$. This is naturally expected since such systems are highly superluminal, that is, there exists no de Hoffman-Teller (1950) shock rest frame where the flow velocities are everywhere parallel to the mean magnetic field (which would correspond to large scale electric fields being zero everywhere). Therefore, relativistic shocks are much less efficient accelerators because particles convect more rapidly away downstream 
from the shock (e.g. Begelman \& Kirk 1990). In oblique, relativistic shocks, $\sigma$, and indeed the efficiency of injection from the thermal particle population, also depend on the ability of turbulence to transport particles perpendicular to the mean downstream field direction (Ellison \& Double 2004; Niemiec \& Ostrowski 2004). This perpendicular transport couples directly to the magnitude $(\delta B / B)^{2}$ and power spectrum of field fluctuations, i.e. the strength of the scattering. Steep spectra $(\sigma \gtrsim 4)$ result unless the ratio of the diffusive mean free paths perpendicular to and parallel to $\mathbf{B}$ is comparable to unity, which defines the Bohm diffusion regime. In summation, for relativistic shocks, the spectral index is sensitive to the obliquity $\Theta_{\mathrm{Bn}, \mathrm{u}}$ of the shock, the nature of the scattering, and the strength of the turbulence or anisotropy of the diffusion. These properties are reviewed in Baring (2004).

Observational vindication of these theoretical predictions is clearly mandated. This is not readily forthcoming, since the only accessible information involves a convolution of shock acceleration and radiation physics. Yet, it is clear, for example in gamma-ray bursts (GRBs), that data taken from the EGRET experiment on the Compton Gamma-Ray Observatory (CGRO) suggest a broad range of spectral indices (Dingus 1995) for the half dozen or so bursts seen at high energies. This population characteristic is commensurate with the expected non-universality of $\sigma$ just discussed. Yet it is important to emphasize that the power-law index is not the only acceleration characteristic germane to the GRB problem: the shapes of the particle distributions at thermal and slightly suprathermal energies are also pertinent. This energy domain samples particle injection or dissipational heating in the shock layer, and is readily probed for electrons by the spectrum of prompt GRB emission by the BATSE instrument on CGRO. Tavani (1996) obtained impressive spectral fits to several bright BATSE bursts using a phenomenological electron distribution and the synchrotron emission mechanism. While there are issues with fitting low energy (i.e. $\lesssim 100 \mathrm{keV}$ ) spectra in about $1 / 3$ of bursts (e.g. Preece et al. 1998) in the synchrotron model, this radiative mechanism still remains the most popular candidate today for prompt burst signals.

Tavani's work was extended recently by Baring \& Braby (2004), who provided additional perspectives, using acceleration theory to underpin a program of spectral fitting of GRB emission using a sum of thermal and non-thermal electron populations. These fits demanded that the preponderance of electrons that are responsible for the prompt emission constitute an intrinsically non-thermal population. That is, the contribution to the overall electron distribution that comes from a Maxwell-Boltzmann distribution is completely dominated by a non-thermal contribution that, to first order, can be approximated by a power-law in energy truncated at some minimum electron Lorentz factor. This requirement of non-thermal dominance strongly contrasts particle distributions obtained from acceleration simulations, as is evident in a host of the references cited on acceleration theory above: the non-thermal particles are drawn directly from a thermal gas, a virtually 
ubiquitous phenomenon. This conflict poses a problem for acceleration scenarios unless (i) radiative efficiencies for electrons in GRBs only become significant at highly superthermal energies, or (ii) shock layer dissipation in relativistic systems can suppress thermalization of electrons. A potential resolution to this dilemma along the lines of option (i) is that strong radiative self-absorption could be acting, in which case the BATSE spectral probe is not actually sampling the thermal electrons. It is also possible that other radiation mechanisms such as Compton scattering, pitch-angle synchrotron, or jitter radiation may prove more germane. Discerning the radiation mechanism(s) operating in bursts is a foremost goal of future research, and will be facilitated by the GLAST mission, with its good sensitivity in the $5 \mathrm{keV}-300 \mathrm{GeV}$ band, in conjunction with NASA's current GRB flagship venture, Swift.

Option (ii) is a conjecture that has no definitive simulational evidence to support it at present. The most comprehensive way to study dissipation and wave generation in collisionless shocks is with PIC simulations, where particle motion and field fluctuations are obtained as solutions of the Newton-Lorentz and Maxwell's equations. Rich in their turbulence information, these have been used extensively in non-relativistic, heliospheric shock applications, and more recently, relativistic PIC codes have blossomed to model shocks in various astrophysical systems. PIC simulation research has largely, but not exclusively, focused on perpendicular shocks, first with Gallant et al.(1992), Hoshino et al. (1992), and then Smolsky \& Usov (1996), Shimada \& Hoshino (2000), Silva et al. (2003), Nishikawa et al. (2003, 2005), Spitkovsky \& Arons (2004), Hededal et al. (2004), Liang \& Nishimura (2004), Medvedev et al. (2005) and Hededal \& Nishikawa (2005). These works have explored pair shocks, ion-doped shocks, Poynting flux-dominated outflows, and low-field systems with dissipation driven by the Weibel instability, in applications such as GRBs and pulsar wind termination shocks. PIC simulations are dynamic in nature, and rarely achieve a timeasymptotic state. Even in the minority of cases where there is some evidence of acceleration beyond true thermalization, none of these works has demonstrated the establishment of an extended power-law that is required in modeling emission from GRBs and active galactic nuclei. This is perhaps due to the severely restricted spatial and temporal scales of the simulations, imposed by their intensive CPU and memory requirements; these limit the modeling of realistic electron-to-proton mass ratios, full exploration of three-dimensional shock physics such as diffusive transport, and addressing the wide range of particle momenta encountered in the shock acceleration process. In particular, it is difficult to establish a broad inertial range for cascading MHD turbulence when the maximum spatial scales in the simulation are not orders of magnitude larger than the principal ion inertial scales. Definitively observing the injection of electrons from a thermal population to establish a truly non-thermal distribution remains a pressing goal of plasma simulations, both for relativistic shocks and, as has been understood for more than two decades, also their non-relativistic cousins. 


\section{Conclusion}

This review is by no means a complete presentation of the topical issues for the shock acceleration problem, but it does offer a fair sampling suitable for motivating interdisciplinary activity. It is clear that several issues could benefit substantially from input from laboratory experimentation on the high energy density physics/astrophysics interface. One key question is whether or not ambient magnetic fields are amplified by both non-relativistic and relativistic shocks beyond standard MHD expectations. If so, is the amplification electrostatic in origin, or is it connected to energetic particles accelerated by the shock? It would be important to discern whether there are differences between high and low Alfvénic Mach number systems, i.e. what role the ambient magnetic field plays in controlling the outcome. Another question concerns whether or not suprathermal electrons and ions can actually be seen, and whether one can identify their origin. It is salient to ascertain if they are diffuse in nature, or if they form coherent beams, both of which are seen at traveling shocks embedded in the solar wind. Also, if acceleration is observed, then identifying the role the highest energy particles have in modifying the shock hydrodynamics and the thermal structure of the shock layer would help solve an outstanding problem that has long been a principal goal within the cosmic ray community. Finally, specifically concerning relativistic systems, it would be desirable to elucidate how the distributions of any accelerated particles seen depend on external quantities such as the field obliquity and speed of the shock, whether thermal electrons can be suppressed relative to accelerated ones, and if there is an identifiable connection with the field turbulence near the shock. These are demanding goals, yet terrestrial experiments are very useful for probing global aspects of shock problems, and in particular for extracting insights into hydrodynamic and MHD behavior. In order to make progress, it is essential to prepare an experimental setup that is as tenuous as possible, to mimic the collisionless (in the Spitzer sense) shock environments offered throughout the cosmos. At this juncture, exciting prospects are on the horizon for this interdisciplinary forum, with contributions to be found in the laboratory, in computer simulations, and in astronomical observations, all of which can benefit from cross-fertilization with each other.

\section{References:}

Allen, G. E., Houck, J. C. \& Sturner, S. J. 2004, in on-line proceedings of the X-ray and Radio Connections workshop, Sante Fe, New Mexico. [http://www.aoc.nrao.edu/events/xraydio]

Amati, E. \& Blasi, P. 2006, M.N.R.A.S., 371, 1251.

Anderson, M. C., Keohane, J. W. \& Rudnick, L. 1995, ApJ, 441, 300.

Bamba, A., Yamazaki, R., Ueno, M. \& Koyama, K. 2003, ApJ, 589, 827. 
Baring, M. G. 1999, in Proc. of the 26th International Cosmic Ray Conference, Vol. IV, p. 5, held in Salt Lake City, Utah, [astro-ph/9910128].

Baring, M. G. 2004, Nuclear Physics B, Proc. Supp., 136, 198.

Baring, M. G. \& Braby, M. L. 2004, ApJ 613, 460.

Baring, M. G., Ellison, D. C., Reynolds, S. P., Grenier, I. A., \& Goret, P. 1999, ApJ, 513, 311.

Baring, M. G., Ellison, D. C., \& Slane, P. O. 2005, Adv. Space. Res., 35, 1041.

Baring, M. G., Ogilvie, K. W., Ellison, D. C. \& Forsyth, R. J. 1997, ApJ, 476, 889.

Baring, M. G. \& Summerlin, E. J. 2006, Astr. Space Sci., in press. [astro-ph/0609407]

Bednarz, J. \& Ostrowski, M. 1998, Phys. Rev. Lett., 80, 3911.

Begelman, M. C. \& Kirk, J. G. 1990, ApJ, 353, 66.

Bell, A. R., 2004, M.N.R.A.S., 353, 550.

Berezhko, E. G., Ksenofontov, L. T. \& Völk, H. J. 2002, ApJ, 395, 943.

Berezhko, E. G., Yelshin, V. K. \& Ksenofontov, L. T. 1996, JETP, 82(1), 1.

Blandford, R. D. \& Eichler, D. 1987, Phys. Rep., 154, 1.

Blasi, P. 2002, Astroparticle Phys., 16, 429.

Borovsky, J. E., Pongratz, M. B., Roussel-Dupre, R. A. \& Tan, T.-H. 1984, ApJ, 280, 802.

Decourchelle, A., Ellison, D. C. \& Ballet, J. 2000, ApJ, 543, L57.

de Hoffman, F. \& Teller, E. 1950, Phys. Rev. D, 80, 692.

Dingus, B. L. 1995, Astr. Space Sci., 231, 187.

Downs, G. S. \& Thompson, A. R. 1972, AJ, 77, 120.

Drake, R. P., et al. 1998, ApJ,500, L157.

Drury, L. O'C. 1983, Rep. Prog. Phys., 46, 973.

Eichler, D. 1984, ApJ, 277, 429.

Ellison, D. C., Baring, M. G. \& Jones, F. C. 1995, ApJ, 453, 873.

Ellison, D. C., Baring, M. G. \& Jones, F. C. 1996, ApJ, 473, 1029.

Ellison, D. C. \& Cassam-Chenaï, G. 2005, ApJ, 632, 920.

Ellison, D. C. \& Double, G. P. 2004, Astroparticle Phys., 22, 323.

Ellison, D. C. \& Eichler, D. 1984, ApJ, 286, 691.

Ellison, D. C., Jones, F. C., \& Reynolds, S. P. 1990, ApJ, 360, 702.

Ellison, D. C., Möbius, E. \& Paschmann, G. 1990 ApJ, 352, 376. 
Ellison, D. C., Slane, P. \& Gaensler, B. M. 2001 ApJ, 563, 191.

Forman, M. A., Jokipii, J. R. \& Owens, A. J. 1974 ApJ, 192, 535.

Gallant, Y. A. \& Achterberg, A. 1999, M.N.R.A.S., 305, L6.

Gallant, Y. A., Hoshino, M., Langdon, A. B., Arons, J., \& Max, C.E. 1992 ApJ, 391, 73.

Ghavamian, P., Rakowski, C. E., Hughes, J. P. \& Williams, T. B. 2003, ApJ, 590, 833.

Hededal,C. B., Haugbolle,T., Frederiksen,J. T. \& Nordlund,A 2004, ApJ, 617, L107.

Hededal, C. B. \& Nishikawa, K.-I. 2005, ApJ, 623, L89.

Hoshino, M., Arons, J., Gallant, Y. A. \& Langdon, A. B. 1992, ApJ, 390, 454.

Hughes, J. P., Rakowski, C. E., \& Decourchelle, A. 2000, ApJ, 543, L61.

Hwang, U., et. al. 2002, ApJ, 581, 110.

Jokipii, J. R. 1987, ApJ, 313, 842.

Jones, F. C. \& Ellison, D. C. 1991, Space Science Rev., 58, 259.

Jones, T. J., Rudnick, L., DeLaney, T. \& Bowden, J. 2003, ApJ, 587, 338.

Kang, Y.-G., et al. 2001, Plasma Phys. Rep., 27, 843.

Kirk, J. G. \& Dendy, R. O. 2001, J. Phys. G., 27, 1589.

Kirk, J. G., Guthmann, A. W., Gallant, Y. A., Achterberg, A. 2000, ApJ, 542, 235.

Lagage, P. O. \& Cesarsky, C. J. 1983, A\&A, 125, 249.

Lebedev, S. V., et al. 2002, ApJ, 564, 113.

Liang, E. P. \& Nishimura, K. 2004, Phys. Rev. Lett., 92, 5005.

Long, K. S., et al. 2003, ApJ, 586, 1162.

Lucek, S. G. \& Bell, A. R. 2000, M.N.R.A.S., 314, 65.

Malkov, M. A. 1997, ApJ, 485, 638.

Medvedev, M. V., et al. 2005, ApJ,618, L75.

Melrose, D. B. 1980, Plasma Astrophysics, Vols. I \& II (Gordon \& Breach, New York)

Niemiec, J., \& Ostrowski, M. 2004, ApJ,610, 851.

Nishikawa, K.-I., et al. 2003, ApJ, 595, 555.

Nishikawa, K.-I., et al. 2005, ApJ, 622, 927.

Preece, R. D., et al. 1998, ApJ, 506, L23.

Remington, B. A., Drake, R. P. \& Ryutov, D. D. 2006, Rev. Mod. Phys. 78, 755.

Reynolds, S. P. \& Ellison, D. C. 1992, ApJ, 399, L75. 
Rosenberg, I. 1970, M.N.R.A.S., 151, 109.

Ryutov, D. D., Remington, B. A., Robey, H. F., Drake, R. P. 2001, Phys. Plasmas, 8, 1804.

Shigemori, K., et al. 2000, ApJ, 533, L159.

Shimada, N. \& Hoshino, M. 2000, ApJ, 543, L67.

Silva, L. O., et al. 2003, ApJ,596, L121.

Smolsky, M. V. \& Usov, V. V. 1996, ApJ, 461, 858.

Spitkovsky, A. \& Arons, J. 2004, ApJ, 603, 669.

Tavani, M. 1996, Phys. Rev. Lett., 76, 3478.

Vietri, M. 1995, ApJ, 453, 883.

Vink, J. \& Laming, J. M. 2003, ApJ, 584, 758.

Vladimirov, A., Ellison, D. C. \& Bykov, A. 2006, ApJ, in press. [astro-ph/0606433]

Völk, H. J., Berezhko, E. G. \& Ksenofontov, L. T. 2005, A\&A, 433, 973.

Woolsey, N. C., Courtois, C. \& Dendy, R. O. 2004, Plasma Phys. Cont. Fus., 46, B397. 